\title{
Zeta functions with respect to general coined quantum walk of periodic graphs
}

\author{
Takashi Komatsu \\ Department of Bioengineering School of Engineering \\ The University of Tokyo \\ Bunkyo, Tokyo, 113-8656, Japan \\ komatsu@coi.t.u-tokyo.ac.jp \\ Norio Konno \\ Department of Applied Mathematics, Faculty of Engineering \\ Yokohama National University \\ Hodogaya, Yokohama 240-8501, Japan \\ konno-norio-bt@ynu.ac.jp \\ Iwao Sato \\ National Institute of Technology, Oyama College \\ Oyama, Tochigi 323-0806, Japan \\ isato@oyama-ct.ac.jp
}

Submitted: Oct 29, 2019; Accepted: Jun 24, 2020; Published: Jul 10, 2020

(C) The authors. Released under the CC BY-ND license (International 4.0).

\begin{abstract}
We define a zeta function of a graph by using the time evolution matrix of a general coined quantum walk on it, and give a determinant expression for the zeta function of a finite graph. Furthermore, we present a determinant expression for the zeta function of an (inifinite) periodic graph.
\end{abstract}

Mathematics Subject Classifications: 60F05, 05C50, 15A15, 05C25

\section{Introduction}

Starting from $p$-adic Selberg zeta functions, Ihara [12] introduced the Ihara zeta functions of graphs. Ihara [12] showed that the reciprocal of the Ihara zeta function of a regular graph is an explicit polynomial. Serre [17] pointed out that the Ihara zeta function is the zeta function of a regular graph. A zeta function of a regular graph $G$ associated to a unitary representation of the fundamental group of $G$ was developed by Sunada 
[18, 19]. Hashimoto [10] treated multivariable zeta functions of bipartite graphs. Bass [1] generalized Ihara's result on the Ihara zeta function of a regular graph to an irregular graph, and showed that its reciprocal is a polynomial.

The Ihara zeta function of a finite graph was extended to an infinite graph in $[1,3$, $6,7,8,9]$, and its determinant expressions were presented. Bass [1] defined the zeta function for a pair of a tree $X$ and a countable group $\Gamma$ which acts discretely on $X$ with quotient being a graph of finite groups. Clair and Mokhtari-Sharghi [3] extended Ihara zeta functions to infinite graphs on which a group $\Gamma$ acts isomorphically and with finite quotient. In [6], Grigorchuk and Żuk defined zeta functions of infinite discrete groups, and of some class of infinite periodic graphs. Guido, Isola and Lapidus [7] defined the Ihara zeta function of a periodic simple graph. Furthermore, Guido, Isola and Lapidus [8] presented a determinant expression for the Ihara zeta function of a periodic graph.

The time evolution matrix of a discrete-time quantum walk in a graph is closely related to the Ihara zeta function of a graph. A discrete-time quantum walk is a quantum analog of the classical random walk on a graph whose state vector is governed by a matrix called the time evolution matrix. Ren et al. [16] gave a relationship between the discrete-time quantum walk and the Ihara zeta function of a graph. Konno and Sato [13] obtained a formula of the characteristic polynomial of the Grover matrix by using the determinant expression for the second weighted zeta function of a graph.

In this paper, we define a zeta function of a periodic graph by using the time evolution matrix of a general coined quantum walk on it, and present its determinant expression. The proof is an analogue of Bass' method [1].

In Section 2, we state a review for the Ihara zeta function of a finite graph and infinite graphs, i.e., a periodic simple graph, a periodic graph. In Section 3, we state about the Grover walk on a graph as a discrete-time quantum walk on a graph. In Section 4, we define a zeta function of a finite graph $G$ by using the time evolution matrix of a general coined quantum walk on $G$, and present its determinant expression. Furthermore, we give an explicit formula for the characteristic polynomial of the time evolution matrix of a general coined quantum walk on $G$, and so present its spectrum. In Section 5, we state the definition of a periodic graph. In Section 6 , we review a determinant for bounded operators acting on an infinite dimensional Hilbert space and belonging to a von Neumann algebra with a finite trace. In Section 7 , we present a determinant expression for the above zeta function of a periodic graph.

\section{The Ihara zeta function of a graph}

All graphs in this paper are assumed to be simple. Let $G$ be a connected graph with vertex set $V(G)$ and edge set $E(G)$, and let $R(G)=\{(u, v),(v, u) \mid u v \in E(G)\}$ be the set of oriented edges (or arcs) $(u, v),(v, u)$ directed oppositely for each edge $u v$ of $G$. For $e=(u, v) \in R(G), u=o(e)$ and $v=t(e)$ are called the origin and the terminal of $e$, respectively. Furthermore, let $e^{-1}=(v, u)$ be the inverse of $e=(u, v)$.

A path $P$ of length $n$ in $G$ is a sequence $P=\left(e_{1}, \cdots, e_{n}\right)$ of $n$ arcs such that $e_{i} \in R(G)$, $t\left(e_{i}\right)=o\left(e_{i+1}\right)(1 \leqslant i \leqslant n-1)$. If $e_{i}=\left(v_{i-1}, v_{i}\right), 1 \leqslant i \leqslant n$, then we also denote $P$ by 
$\left(v_{0}, v_{1}, \cdots, v_{n}\right)$. Set $|P|=n, o(P)=o\left(e_{1}\right)$ and $t(P)=t\left(e_{n}\right)$. Also, $P$ is called an $(o(P), t(P))$-path. A $(v, w)$-path is called a $v$-closed path if $v=w$. The inverse of a closed path $C=\left(e_{1}, \cdots, e_{n}\right)$ is the closed path $C^{-1}=\left(e_{n}^{-1}, \cdots, e_{1}^{-1}\right)$.

We say that a path $P=\left(e_{1}, \cdots, e_{n}\right)$ has a backtracking if $e_{i+1}^{-1}=e_{i}$ for some $i(1 \leqslant i \leqslant$ $n-1)$. A path without backtracking is called proper. Let $B^{r}$ be the closed path obtained by going $r$ times around a closed path $B$. Such a closed path is called a multiple of $B$. Multiples of a closed path without backtracking may have a backtracking. Such a closed path is said to have a tail. If its length is $n$, then the closed path can be written as

$$
\left(e_{1}, \cdots, e_{k}, f_{1}, f_{2}, \cdots, f_{n-2 k}, e_{k}^{-1}, \cdots, e_{1}^{-1}\right),
$$

where $\left(f_{1}, f_{2}, \cdots, f_{n-2 k}\right)$ is a closed path. A closed path is called reduced if $C$ has no backtracking nor tail. Furthermore, a closed path $C$ is primitive if it is not a multiple of a strictly shorter closed path.

We introduce an equivalence relation between closed paths. Two closed paths $C_{1}=$ $\left(e_{1}, \cdots, e_{m}\right)$ and $C_{2}=\left(f_{1}, \cdots, f_{m}\right)$ are called equivalent if there exists an integer $k$ such that $f_{j}=e_{j+k}$ for all $j$, where the subscripts are read modulo $n$. The inverse of $C$ is not equivalent to $C$ if $|C| \geqslant 3$. Let $[C]$ be the equivalence class which contains a closed path $C$. Also, $[C]$ is called a cycle.

Let $\mathcal{P}$ be the set of primitive, reduced cycles of $G$. Also, primitive, reduced cycles are called prime cycles. Note that each equivalence class of primitive, reduced closed paths of a graph $G$ passing through a vertex $v$ of $G$ corresponds to a unique conjugacy class of the fundamental group $\pi_{1}(G, v)$ of $G$ at $v$.

The Ihara zeta function of a graph $G$ is a function of a complex variable $u$ with $|u|$ sufficiently small, defined by

$$
\mathbf{Z}(G, u)=\mathbf{Z}_{G}(u)=\prod_{[C] \in \mathcal{P}}\left(1-u^{|C|}\right)^{-1},
$$

where $[C]$ runs over all prime cycles of $G$.

Let $G$ be a connected graph with $n$ vertices $v_{1}, \cdots, v_{n}$. The adjacency matrix $\mathbf{A}=$ $\mathbf{A}(G)=\left(a_{i j}\right)$ is the square matrix such that $a_{i j}=1$ if $v_{i}$ and $v_{j}$ are adjacent, and $a_{i j}=0$ otherwise. The degree of a vertex $v_{i}$ of $G$ is defined by $\operatorname{deg} v_{i}=\operatorname{deg}{ }_{G} v_{i}=\mid\left\{v_{j} \mid v_{i} v_{j} \in\right.$ $E(G)\} \mid$. If $\operatorname{deg}_{G} v=k$ (constant) for each $v \in V(G)$, then $G$ is called $k$-regular.

Theorem 1 (Bass). Let $G$ be a connected graph. Then the reciprocal of the Ihara zeta function of $G$ is given by

$$
\mathbf{Z}(G, u)^{-1}=\left(1-u^{2}\right)^{r-1} \operatorname{det}\left(\mathbf{I}-u \mathbf{A}(G)+u^{2}(\mathbf{D}-\mathbf{I})\right),
$$

where $r$ is the Betti number of $G$, and $\mathbf{D}=\left(d_{i j}\right)$ is the diagonal matrix with $d_{i i}=\operatorname{deg} v_{i}$ and $d_{i j}=0, i \neq j,\left(V(G)=\left\{v_{1}, \cdots, v_{n}\right\}\right)$.

Let $G=(V(G), E(G))$ be a countable simple graph, and let $\Gamma$ be a countable discrete subgroup of automorphisms of $G$, which acts freely on $G$, and with finite quotient $G / \Gamma$. 
The graph $G$ is called a periodic graph. Then the Ihara zeta function of a periodic simple graph is defined as follows:

$$
\mathbf{Z}_{G, \Gamma}(u)=\prod_{[C]_{\Gamma} \in[\mathcal{P}]_{\Gamma}}\left(1-u^{|C|}\right)^{-1 /\left|\Gamma_{[C]}\right|}
$$

where $\Gamma_{[C]}$ is the stabilizer of $[C]$ in $\Gamma$, and $[C]_{\Gamma}$ runs over all $\Gamma$-equivalence classes of prime cycles in $G$.

Guido, Isola and Lapidus [7] presented a determinant expression for the Ihara zeta function of a periodic simple graph.

Theorem 2 (Guido, Isola and Lapidus). For a periodic simple graph $G$,

$$
\mathbf{Z}_{G, \Gamma}(u)=\left(1-u^{2}\right)^{-(m-n)} \operatorname{det}_{\Gamma}\left(\mathbf{I}-u \mathbf{A}(G)+(\mathbf{D}-\mathbf{I}) u^{2}\right)^{-1},
$$

where $\operatorname{det}_{\Gamma}$ is a determinant for bounded operators belonging to a von Neumann algebra with a finite trace.

Guido, Isola and Lapidus [8] presented a determinant expression for the Ihara zeta function of a periodic graph $G$ and a countable discrete subgroup $\Gamma$ of aoutomorphisms of $G$ which acts discretely without inversions, and with bounded covolume.

Theorem 3 (Guido, Isola and Lapidus). For a periodic graph $G$,

$$
\mathbf{Z}_{G, \Gamma}(u)^{-1}=\left(1-u^{2}\right)^{\chi^{(2)}(G)} \operatorname{det}_{\Gamma}(\Delta(u)),
$$

where $\chi^{(2)}(G)$ is the $L^{2}$-Euler characteristic of $(G, \Gamma)$ (see [2]), and $\Delta(u)=\mathbf{I}-u \mathbf{A}+$ $u^{2}(\mathbf{D}-\mathbf{I})$.

\section{The Grover walk on a graph}

Let $G$ be a connected graph with $n$ vertices and $m$ edges, $V(G)=\left\{v_{1}, \ldots, v_{n}\right\}$ and $R(G)=\left\{e_{1}, \ldots, e_{m}, e_{1}^{-1}, \ldots, e_{m}^{-1}\right\}$. Set $d_{j}=d_{v_{j}}=\operatorname{deg} v_{j}$ for $i=1, \ldots, n$. The Grover matrix $\mathbf{U}=\mathbf{U}(G)=\left(U_{e f}\right)_{e, f \in R(G)}$ of $G$ is defined by

$$
U_{e f}= \begin{cases}2 / d_{t(f)}\left(=2 / d_{o(e)}\right) & \text { if } t(f)=o(e) \text { and } f \neq e^{-1} \\ 2 / d_{t(f)}-1 & \text { if } f=e^{-1} \\ 0 & \text { otherwise. }\end{cases}
$$

The discrete-time quantum walk with the matrix $\mathbf{U}$ as a time evolution matrix is called the Grover walk on $G$.

Let $G$ be a connected graph with $n$ vertices and $m$ edges. Then the $n \times n$ matrix $\mathbf{T}(G)=\left(T_{u v}\right)_{u, v \in V(G)}$ is given as follows:

$$
T_{u v}= \begin{cases}1 /\left(\operatorname{deg}_{G} u\right) & \text { if }(u, v) \in R(G), \\ 0 & \text { otherwise. }\end{cases}
$$

Note that the matrix $\mathbf{T}(G)$ is the transition matrix of the simple random walk on $G$. 
Theorem 4 (Konno and Sato). Let $G$ be a connected graph with $n$ vertices $v_{1}, \ldots, v_{n}$ and $m$ edges. Then the characteristic polynomial for the Grover matrix $\mathbf{U}$ of $G$ is given by

$$
\begin{aligned}
\operatorname{det}\left(\lambda \mathbf{I}_{2 m}-\mathbf{U}\right) & =\left(\lambda^{2}-1\right)^{m-n} \operatorname{det}\left(\left(\lambda^{2}+1\right) \mathbf{I}_{n}-2 \lambda \mathbf{T}(G)\right) \\
& =\frac{\left(\lambda^{2}-1\right)^{m-n} \operatorname{det}\left(\left(\lambda^{2}+1\right) \mathbf{D}-2 \lambda \mathbf{A}(G)\right)}{d_{v_{1}} \cdots d_{v_{n}}}
\end{aligned}
$$

From this Theorem, the spectra of the Grover matrix on a graph is obtained by means of those of $\mathbf{T}(G)$ (see [4]). Let $\operatorname{Spec}(\mathbf{F})$ be the spectra of a square matrix $\mathbf{F}$.

Corollary 5 (Emms, Hancock, Severini and Wilson). Let $G$ be a connected graph with $n$ vertices and $m$ edges. The Grover matrix $\mathbf{U}$ has $2 n$ eigenvalues of the form

$$
\lambda=\lambda_{T} \pm i \sqrt{1-\lambda_{T}^{2}}
$$

where $\lambda_{T}$ is an eigenvalue of the matrix $\mathbf{T}(G)$. The remaining $2(m-n)$ eigenvalues of $\mathbf{U}$ are \pm 1 with equal multiplicities.

\section{Spectra for the time evolution matrix of a general coined quantum walk on a graph}

We consider a generalization of a coined quantum walk on a graph. We replace the coin operator $\mathbf{C}$ of a coined quantum walk with unitary matrix with two spectra which are distinct from \pm 1 .

For a given connected graph $G$ with $n$ vertices and $m$ edges, let $\mathbf{d}: \ell^{2}(V(G)) \longrightarrow$ $\ell^{2}(R(G))$ such that

$$
\mathbf{d d}^{*}=\mathbf{I}_{q},
$$

and let $\mathbf{S}=\left(S_{e f}\right)_{e, f \in R(G)}$ be the $2 m \times 2 m$ matrix defined by

$$
S_{e f}= \begin{cases}1 & \text { if } f=e^{-1} \\ 0 & \text { otherwise }\end{cases}
$$

Furthermore, let

$$
\mathbf{C}=a \mathbf{d}^{*} \mathbf{d}+b\left(\mathbf{I}_{2 m}-\mathbf{d}^{*} \mathbf{d}\right)
$$

and $\mathbf{U}=\mathbf{S C}($ see [11]). Note that $q=\operatorname{dim} \operatorname{ker}(a-\mathbf{C})$. A discrete-time quantum walk on $G$ with $\mathbf{U}$ as a time evolution matrix is called a general coined quantum walk on $G$. Then we define a zeta function of $G$ by using $\mathbf{U}$ as follows:

$$
\zeta(G, u)=\operatorname{det}\left(\mathbf{I}_{2 m}-u \mathbf{U}\right)^{-1}=\operatorname{det}\left(\mathbf{I}_{2 m}-u \mathbf{S}\left(a \mathbf{d}^{*} \mathbf{d}+b\left(\mathbf{I}_{2 m}-\mathbf{d}^{*} \mathbf{d}\right)\right)\right)^{-1} .
$$

Now, we have the following result. 
Theorem 6. Let $G$ be a connected graph $n$ vertices and $m$ edges, $\mathbf{U}=\mathbf{S C}$ the time evolution matrix of a general coined quantum walk on $G$. Suppose that $\sigma(\mathbf{C})=\{a, b\}$. Set $q=\operatorname{dim} \operatorname{ker}(a-\mathbf{C})$. Then, for the unitary matrix $\mathbf{U}=\mathbf{S C}$, we have

$$
\zeta(G, u)=\left(1-b^{2} u^{2}\right)^{m-q} \operatorname{det}\left(\left(1-a b u^{2}\right) \mathbf{I}_{n}-c u \mathbf{d} \mathbf{S d}^{*}\right), c=a-b .
$$

Proof. At first, we have

$$
\begin{aligned}
& \zeta(G, u)=\operatorname{det}\left(\mathbf{I}_{2 m}-u \mathbf{U}\right)=\operatorname{det}\left(\mathbf{I}_{2 m}-u \mathbf{S C}\right) \\
= & \operatorname{det}\left(\mathbf{I}_{2 m}-u \mathbf{S}\left(a \mathbf{d}^{*} \mathbf{d}+b\left(\mathbf{I}_{2 m}-\mathbf{d}^{*} \mathbf{d}\right)\right)\right) \\
= & \left.\operatorname{det}\left(\mathbf{I}_{2 m}-u \mathbf{S}\left((a-b) \mathbf{d}^{*} \mathbf{d}+b \mathbf{I}_{2 m}\right)\right)\right) \\
= & \operatorname{det}\left(\mathbf{I}_{2 m}-b u \mathbf{S}-c u \mathbf{S} \mathbf{d}^{*} \mathbf{d}\right) \\
= & \operatorname{det}\left(\mathbf{I}_{2 m}-c u \mathbf{S} \mathbf{d}^{*} \mathbf{d}\left(\mathbf{I}_{2 m}-b u \mathbf{S}\right)^{-1}\right) \operatorname{det}\left(\mathbf{I}_{2 m}-b u \mathbf{S}\right) .
\end{aligned}
$$

But, if $\mathbf{A}$ and $\mathbf{B}$ are an $m \times n$ matrix and an $n \times m$ matrix, respectively, then we have

$$
\operatorname{det}\left(\mathbf{I}_{m}-\mathbf{A B}\right)=\operatorname{det}\left(\mathbf{I}_{n}-\mathbf{B A}\right) .
$$

Thus, we have

$$
\operatorname{det}\left(\mathbf{I}_{2 m}-u \mathbf{U}\right)=\operatorname{det}\left(\mathbf{I}_{2 m}-u \mathbf{S C}\right)=\operatorname{det}\left(\mathbf{I}_{n}-c u \mathbf{d}\left(\mathbf{I}_{2 m}-b u \mathbf{S}\right)^{-1} \mathbf{S d}^{*}\right) \operatorname{det}\left(\mathbf{I}_{2 m}-b u \mathbf{S}\right) .
$$

But, we have

$$
\operatorname{det}\left(\mathbf{I}_{2 m}-b u \mathbf{S}\right)=\left(1-b^{2} u^{2}\right)^{m} .
$$

Furthermore, we have

$$
\left(\mathbf{I}_{2 m}-b u \mathbf{S}\right)^{-1}=\frac{1}{1-b^{2} u^{2}}\left(\mathbf{I}_{2 m}+u \mathbf{S}\right) .
$$

Therefore, it follows that

$$
\begin{aligned}
& \operatorname{det}\left(\mathbf{I}_{2 m}-u \mathbf{U}\right) \\
& =\left(1-b^{2} u^{2}\right)^{m} \operatorname{det}\left(\mathbf{I}_{2 m}-\frac{c u}{1-b^{2} u^{2}} \mathbf{S d}^{*} \mathbf{d}\left(\mathbf{I}_{2 m}+b u \mathbf{S}\right)\right) \\
& =\left(1-b^{2} u^{2}\right)^{m} \operatorname{det}\left(\mathbf{I}_{q}-\frac{c u}{1-b^{2} u^{2}} \mathbf{d}\left(\mathbf{I}_{2 m}+b u \mathbf{S}\right) \mathbf{S d}^{*}\right) \\
& =\left(1-b^{2} u^{2}\right)^{m-n} \operatorname{det}\left(\left(1-b^{2} u^{2}\right) \mathbf{I}_{q}-c u \mathbf{d} \mathbf{S d} \mathbf{d}^{*}-b c u^{2} \mathbf{d} \mathbf{S}^{2} \mathbf{d}^{*}\right) \\
& =\left(1-b^{2} u^{2}\right)^{m-n} \operatorname{det}\left(\left(1-b^{2} u^{2}\right) \mathbf{I}_{q}-c u \mathbf{d} \mathbf{S} d^{*}-b c u^{2} \mathbf{I}_{n}\right) \\
& =\left(1-b^{2} u^{2}\right)^{m-n} \operatorname{det}\left(\left(1-a b u^{2}\right) \mathbf{I}_{q}-c u \mathbf{d} \mathbf{S d}^{*}\right) .
\end{aligned}
$$


Corollary 7. Let $G$ be a connected with $n$ vertices and $m$ edges. Then, for the unitary matrix $\mathbf{U}=\mathbf{S C}$, we have

$$
\operatorname{det}\left(\lambda \mathbf{I}_{2 m}-u \mathbf{U}\right)=\left(\lambda^{2}-b^{2}\right)^{m-q} \operatorname{det}\left(\left(\lambda^{2}-a b\right) \mathbf{I}_{q}-c \lambda \mathbf{d} \mathbf{S} \mathbf{d}^{*}\right),
$$

where $q=\operatorname{dim} \operatorname{ker}(1-\mathbf{C})$.

Proof. Let $u=1 / \lambda$. Then, by Theorem 6 , we have

$$
\operatorname{det}\left(\mathbf{I}_{2 m}-1 / \lambda \mathbf{U}\right)=\left(1-b^{2} / \lambda^{2}\right)^{m-q} \operatorname{det}\left(\left(1-a b / \lambda^{2}\right) \mathbf{I}_{q}-c / \lambda \mathbf{d} \mathbf{S d}^{*}\right),
$$

and so,

$$
\operatorname{det}\left(\lambda \mathbf{I}_{2 m}-\mathbf{U}\right)=\left(\lambda^{2}-b^{2}\right)^{m-q} \operatorname{det}\left(\left(\lambda^{2}-a b\right) \mathbf{I}_{q}-c \lambda \mathbf{d} \mathbf{S} \mathbf{d}^{*}\right) .
$$

By Corollary 7, the following result holds.

Corollary 8. Let $G$ be a connected with $n$ vertices and $m$ edges. Then, the spectra of the unitary matrix $\mathbf{U}=\mathbf{S C}$ are given as follows:

1. $2 q$ eigenvalues:

$$
\lambda=\frac{c \mu \pm \sqrt{c^{2} \mu^{2}+4 a b}}{2}, \mu \in \operatorname{Spec}\left(\mathbf{d S d}^{*}\right)
$$

2. The rest eigenvalues are $\pm b$ with the same multiplicity $m-q$.

Proof. By Corollary 7, we have

$$
\begin{aligned}
& \operatorname{det}\left(\lambda \mathbf{I}_{2 m}-\mathbf{U}\right) \\
= & \left(\lambda^{2}-b^{2}\right)^{m-q} \prod_{\mu \in \operatorname{Spec}\left(\mathbf{d S d}^{*}\right)}\left(\lambda^{2}-c \mu \lambda-a b\right) .
\end{aligned}
$$

Solving $\lambda^{2}-2 \mu \lambda+1=0$, we obtain

$$
\lambda=\frac{c \mu \pm \sqrt{c^{2} \mu^{2}+4 a b}}{2} .
$$

The result follows.

\section{$5 \quad$ Periodic graphs}

Let $G=(V(G), E(G))$ be a simple graph. Assume that $G$ is countable $(V(G)$ and $E(G)$ are countable), and with bounded degree, i.e., $d=\sup _{v \in V(G)} \operatorname{deg} v<\infty$. Let $\Gamma$ be a countable discrete subgroup of automorphisms of $G$, which acts

1. without inversions: $\gamma(e) \neq e^{-1}$ for any $\gamma \in \Gamma, e \in R(G)$,

2. discretely: $\Gamma_{v}=\{\gamma \in \Gamma \mid \gamma v=v\}$ is finite for any $v \in V(G)$, 
3. with bounded covolume: $\operatorname{vol}(G / \Gamma):=\sum_{v \in \mathcal{F}_{0}} \frac{1}{\left|\Gamma_{v}\right|}<\infty$, where $\mathcal{F}_{0} \subset V(G)$ contains exactly one representative for each equivalence class in $V(G / \Gamma)$.

Then $G$ is called a periodic graph with a countable discrete subgroup $\Gamma$ of Aut $G$. Note that the third condition is equivalent to the following condition:

$$
\operatorname{vol}(R(G) / \Gamma):=\sum_{e \in \mathcal{F}_{1}} \frac{1}{\left|\Gamma_{e}\right|}<\infty,
$$

where a subset $\mathcal{F}_{1}$ of $R(G)$ contains exactly one representative for each equivalence class in $R(G / \Gamma)$.

Let $\ell^{2}(V(G))$ be the Hilbert space of functions $f: V(G) \longrightarrow \mathbb{C}$ such that $\|f\|:=$ $\sum_{v \in V(G)}|f(v)|^{2}<\infty$. We define the left regular representation $\lambda_{0}$ of $\Gamma$ on $\ell^{2}(V(G))$ as follows:

$$
\left(\lambda_{0}(\gamma) f\right)(x)=f\left(\gamma^{-1} x\right), \gamma \in \Gamma, f \in \ell^{2}(V(G)), x \in V(G) .
$$

We state the definition of a von Neumann algebra. Let $H$ be a separable complex Hilbert space, and let $\mathcal{B}(H)$ denote the $\mathbf{C}^{*}$-algebra of bounded linear operators on $H$. For a subset $M \subset \mathcal{B}(H)$, the commutant of $M$ is $M^{\prime}=\{T \in \mathcal{B}(H) \mid S T=T S, \forall S \in M\}$. Then a von Neumann algebra is a subalgebra $\mathcal{A} \leqslant \mathcal{B}(H)$ such that $\mathcal{A}^{\prime \prime}=\mathcal{A}$. It is known that a determinant is defined for a suitable class of operators in a von Neumann algebra with a finite trace (see $[5,7])$.

For the Hilbert space $\ell^{2}(V(G))$, we consider a von Neumann algebra. Let $\mathcal{B}\left(\ell^{2}(V(G))\right)$ be the $\mathbb{C}^{*}$-algebra of bounded linear operators on $\ell^{2}(V(G))$. A bounded linear operator $A$ of $\mathcal{B}\left(\ell^{2}(V(G))\right)$ acts on $\ell^{2}(V(G))$ by

$$
A(f)(v)=\sum_{w \in V(G)} A(v, w) f(w), v \in V(G), f \in \ell^{2}(V(G)) .
$$

Then the von Neumann algebra $\mathcal{N}_{0}(G, \Gamma)$ of bounded operators on $\ell^{2}(V(G))$ commuting with the action of $\Gamma$ is defined as follows:

$$
\mathcal{N}_{0}(G, \Gamma)=\left\{\lambda_{0}(\gamma) \mid \gamma \in \Gamma\right\}^{\prime}=\left\{T \in \mathcal{B}\left(\ell^{2}(V(G))\right) \mid \lambda_{0}(\gamma) T=T \lambda_{0}(\gamma), \forall \gamma \in \Gamma\right\}
$$

The von Neumann algebra $\mathcal{N}_{0}(G, \Gamma)$ inherits a trace by

$$
\operatorname{Tr}_{\Gamma}(A)=\sum_{x \in \mathcal{F}_{0}} \frac{1}{\left|\Gamma_{x}\right|} A(x, x), A \in \mathcal{N}_{0}(G, \Gamma) .
$$

Let the adjacency matrix $\mathbf{A}=\mathbf{A}(G)$ of $G$ be defined by

$$
(\mathbf{A} f)(v)=\sum_{(v, w) \in R(G)} f(w), f \in \ell^{2}(V(G)) .
$$

By $[14,15]$, we have

$$
\|\mathbf{A}\| \leqslant d=\sup _{v \in V(G)} \operatorname{deg}_{G} v<\infty
$$


and so $\mathbf{A} \in \mathcal{N}_{0}(G, \Gamma)$.

Similarly to $\ell^{2}(V(G))$, we consider the Hilbert space $\ell^{2}(R(G))$ of functions $f: R(G) \rightarrow$ $\mathbb{C}$ such that $\|\omega\|:=\sum_{e \in R(G)}|\omega(e)|^{2}<\infty$. We define the left regular representation $\lambda_{1}$ of $\Gamma$ on $\ell^{2}(R(G))$ as follows:

$$
\left(\lambda_{1}(\gamma) \omega\right)(e)=\omega\left(\gamma^{-1} e\right), \gamma \in \Gamma, \omega \in \ell^{2}(R(G)), e \in R(G)
$$

Then the von Neumann algebra $\mathcal{N}_{1}(G, \Gamma)=\left\{\lambda_{1}(\gamma) \mid \gamma \in \Gamma\right\}^{\prime}$ of bounded operators on $\ell^{2}(R(G))$ commuting with the action of $\Gamma$, inherits a trace by

$$
\operatorname{Tr}_{\Gamma}(A)=\sum_{e \in \mathcal{F}_{1}} \frac{1}{\left|\Gamma_{e}\right|} A(e, e), A \in \mathcal{N}_{1}(G, \Gamma) .
$$

\section{An analytic determinant for von Neumann algebras with a finite trace}

In an excellent paper [5], Fuglede and Kadison defined a positive-valued determinant for a von Neumann algebra with trivial center and finite trace $\tau$. For an invertible operator $A$ with polar decomposition $A=U H$, the Fuglede-Kadison determinant of $A$ is defined by

$$
\operatorname{Det}(A)=\exp \circ \tau \circ \log H,
$$

where $\log H$ may be defined via functional calculus.

Guido, Isola and Lapidus [7] extended the Fuglede-Kadison determinant to a determinant which is an analytic function. Let $(\mathcal{A}, \tau)$ be a von Neumann algebra with a finite trace $\tau$. Then, for $A \in \mathcal{A}$, let

$$
\operatorname{det}_{\tau}(A)=\exp \circ \tau \circ \log A
$$

where

$$
\log (A):=\frac{1}{2 \pi i} \int_{\Lambda} \log \lambda(\lambda-A)^{-1} d \lambda,
$$

and $\Lambda$ is the boundary of a connected, simply connected region $\Omega$ containing the spectrum $\sigma(A)$ of $A$. Then the following lemma holds (see Lemma 5.1 of [7]).

Lemma 9 (Guido, Isola and Lapidus). Let $\mathcal{A}, \Omega, \Gamma$ be as above, and $\phi, \psi$ two branches of the logarithm such that both domains contain $\Omega$. Then

$$
\exp \circ \tau \circ \phi(A)=\exp \circ \tau \circ \psi(A)
$$

Next, we consider a determinant on some subset of $\mathcal{A}$. Let $(\mathcal{A}, \tau)$ be a von Neumann algebra with a finite trace, and $\mathcal{A}_{0}=\{A \in \mathcal{A} \mid 0 \notin$ conv $\sigma(A)\}$, where conv $\sigma(A)$ is the convex hull of $\sigma(A)$. For any $A \in \mathcal{A}_{0}$, we set

$$
\operatorname{det}_{\tau}(A)=\exp \circ \tau \circ\left(\frac{1}{2 \pi i} \int_{\Lambda} \log \lambda(\lambda-A)^{-1} d \lambda\right),
$$


where $\Lambda$ is the boundary of a connected, simply connected region $\Omega$ containing the spectrum conv $\sigma(A)$, and log is a branch of the logarithm whose domain contains $\Omega$. Then the above determinant is well-defined and analytic on $\mathcal{A}_{0}$ (see Corollary 5.3 of [7]). Furthermore, Guido, Isola and Lapidus of $[7,8]$ showed that $\operatorname{det}_{\tau}$ has the following properties.

Proposition 10 (Guido, Isola and Lapidus). Let $(\mathcal{A}, \tau)$ be a von Neumann algebra with a finite trace, $A \in \mathcal{A}_{0}$. Then

1. $\operatorname{det}_{\tau}(z A)=z^{\tau(I)} \operatorname{det}_{\tau}(A)$ for any $z \in \mathbb{C} \backslash\{0\}$.

2. If $A$ is normal, and $A=U H$ is its polar decomposition,

$$
\operatorname{det}_{\tau}(A)=\operatorname{det}_{\tau}(U) \operatorname{det}_{\tau}(H) .
$$

3. If $A$ is positive, $\operatorname{det}_{\tau}(A)=\operatorname{Det}(A)$, where $\operatorname{Det}(A)$ is the Fuglede-Kadison determinant of $A$.

Proposition 11 (Guido, Isola and Lapidus). Let $(\mathcal{A}, \tau)$ be a von Neumann algebra with a finite trace. Then

1. For $A, B \in \mathcal{A}$ and sufficiently small $u \in \mathbb{C}$,

$$
\operatorname{det}_{\tau}((I+u A)(I+u B))=\operatorname{det}_{\tau}(I+u A) \operatorname{det}_{\tau}(I+u B) .
$$

2. If $A \in \mathcal{A}$ has a bounded inverse, and $T \in \mathcal{A}_{0}$, then

$$
\operatorname{det}_{\tau}\left(A T A^{-1}\right)=\operatorname{det}_{\tau}(T)
$$

3. If

$$
T=\left[\begin{array}{cc}
T_{11} & T_{12} \\
0 & T_{22}
\end{array}\right] \in \operatorname{Mat}_{2}(\mathcal{A})
$$

with $T_{i i} \in \mathcal{A}$ such that $\sigma\left(T_{i i}\right) \subset B_{1}(1):=\{z \in \mathbb{C}|| z-1 \mid<1\}$ for $i=1,2$, then

$$
\operatorname{det}_{\tau}(T)=\operatorname{det}_{\tau}\left(T_{11}\right) \operatorname{det}_{\tau}\left(T_{22}\right) .
$$

Corollary 12 (Guido, Isola and Lapidus). Let $\Gamma$ be a discrete group, $\pi_{1}, \pi_{2}$ unitary representations of $\Gamma, \tau_{1}, \tau_{2}$ finite traces on $\pi_{1}(\Gamma)^{\prime}$ and $\pi_{2}(\Gamma)^{\prime}$, respectively. Let $\pi=\pi_{1} \oplus \pi_{2}$, $\tau=\tau_{1}+\tau_{2}$ and $T=\left[\begin{array}{cc}T_{11} & T_{12} \\ 0 & T_{22}\end{array}\right] \in \pi(\Gamma)^{\prime}$, with $\sigma\left(T_{i i}\right) \subset B_{1}(1):=\{z \in \mathbf{C}|| z-1 \mid<1\}$ for $i=1,2$, then

$$
\operatorname{det}_{\tau}(T)=\operatorname{det}_{\tau_{1}}\left(T_{11}\right) \operatorname{det}_{\tau_{2}}\left(T_{22}\right) .
$$




\section{A zeta function with respect to a general coined quantum walk of an infinite periodic graph}

We define a zeta function with respect to a general coined quantum walk of an infinite periodic graph.

Let $G$ be a periodic graph with a countable discrete subgroup $\Gamma$ of Aut $G$. Moreover, let

$$
\mathbf{I}_{V}=I d_{\ell^{2}(V(G))}, \mathbf{I}_{R}=I d_{\ell^{2}(R(G))} .
$$

Then, let $\mathbf{d}: \ell^{2}(V(G)) \longrightarrow \ell^{2}(R(G))$ such that

$$
\mathbf{d d}^{*}=\mathbf{I}_{V}
$$

Furthermore, let

$$
\mathbf{C}=a \mathbf{d}^{*} \mathbf{d}+b\left(\mathbf{I}_{R}-\mathbf{d}^{*} \mathbf{d}\right)
$$

and $\mathbf{U}=\mathbf{S C}$, where $\mathbf{S}$ is the operator on $\ell^{2}(R(G))$ such that

$$
(\mathbf{S} \omega)(e)=\omega\left(e^{-1}\right), \omega \in \ell^{2}(R(G)), e \in R(G) .
$$

Now, we consider the following determinant:

$$
\operatorname{det}_{\Gamma}(B)=\exp \circ \operatorname{Tr}_{\Gamma} \circ \log B
$$

for $B \in \mathcal{N}_{1}(G, \Gamma)_{0}$. Then a zeta function with respect to a general coined quantum walk of $G$ is defined as follows:

$$
\zeta(G, \Gamma, u)=\operatorname{det}_{\Gamma}\left(\mathbf{I}_{R}-u \mathbf{U}\right)^{-1}=\operatorname{det}_{\Gamma}\left(\mathbf{I}_{R}-u \mathbf{S}\left(a \mathbf{d}^{*} \mathbf{d}+b\left(\mathbf{I}_{R}-\mathbf{d}^{*} \mathbf{d}\right)\right)\right)^{-1},
$$

where $u \in \mathbb{C}$ are sufficiently small so that the infinite product converges.

Then we have the following result.

Theorem 13. Let $G$ be a periodic graph with a countable discrete subgroup $\Gamma$ of Aut $G$. Then

$$
\zeta(G, \Gamma, u)=\left(1-b^{2} u^{2}\right)^{\operatorname{Tr}_{\Gamma}\left(\mathbf{I}_{V}\right)-\frac{1}{2} \operatorname{Tr}_{\Gamma}\left(\mathbf{I}_{R}\right)} \operatorname{det}{ }_{\Gamma}\left(\left(1-a b u^{2}\right) \mathbf{I}_{V}-c u \mathbf{d} \mathbf{S d}^{*}\right),
$$

where $\operatorname{Tr}_{\Gamma}\left(\mathbf{I}_{R}\right)=\sum_{e \in \mathcal{F}_{1}} \frac{1}{\left|\Gamma_{e}\right|}$ and $\operatorname{Tr}_{\Gamma}\left(\mathbf{I}_{V}\right)=\sum_{v \in \mathcal{F}_{0}} \frac{1}{\left|\Gamma_{v}\right|}($ see [2]).

Proof. The argument is an analogue of the method of Bass [1].

Let $G$ be a periodic graph with a countable discrete subgroup $\Gamma$ of $A$ ut $G$.

Now we consider the direct sum of the unitary representations $\lambda_{0}$ and $\lambda_{1}: \lambda(\gamma):=$ $\lambda_{0}(\gamma) \oplus \lambda_{1}(\gamma) \in \mathcal{B}\left(\ell^{2}(V(G)) \oplus \ell^{2}(R(G))\right)$. Then the von Neumann algebra $\lambda(\Gamma)^{\prime}:=\{S \in$ $\left.\mathcal{B}\left(\ell^{2}(V(G)) \oplus \ell^{2}(R(G))\right) \mid S \lambda(\gamma)=\lambda(\gamma) S, \gamma \in \Gamma\right\}$ consists of operators

$$
S=\left[\begin{array}{ll}
S_{00} & S_{01} \\
S_{10} & S_{11}
\end{array}\right],
$$


where $S_{i j} \lambda_{j}(\gamma)=\lambda_{i}(\gamma) S_{i j}, \gamma \in \Gamma, i, j=0,1$, so that $S_{i i} \in \Lambda_{i} \equiv \mathcal{N}_{i}(G, \Gamma), i=0,1$. Thus, $\lambda(\Gamma)^{\prime}$ inherits a trace given by

$$
\operatorname{Tr}_{\Gamma}\left[\begin{array}{ll}
S_{00} & S_{01} \\
S_{10} & S_{11}
\end{array}\right]:=\operatorname{Tr}_{\Gamma}\left(S_{00}\right)+\operatorname{Tr}_{\Gamma}\left(S_{11}\right)
$$

We introduce two operators as follows:

$$
\mathbf{L}=\left[\begin{array}{cc}
\left(1-b^{2} u^{2}\right) \mathbf{I}_{V} & -c \mathbf{d}-b c u \mathbf{d} \mathbf{S} \\
0 & \mathbf{I}_{R}
\end{array}\right], \mathbf{M}=\left[\begin{array}{cc}
\mathbf{I}_{V} & c \mathbf{d}+b c u \mathbf{d} \mathbf{S} \\
u \mathbf{S d}^{*} & \left(1-b^{2} u^{2}\right) \mathbf{I}_{R}
\end{array}\right]
$$

where $c=a-b$. Then we have

$$
\begin{aligned}
\mathbf{L M} & =\left[\begin{array}{cc}
\left(1-b^{2} u^{2}\right) \mathbf{I}_{V}-c u \mathbf{d} \mathbf{S} d^{*}-b c u^{2} \mathbf{d} \mathbf{S}^{2} \mathbf{d}^{*} & 0 \\
u \mathbf{S} \mathbf{d}^{*} & \left(1-b^{2} u^{2}\right) \mathbf{I}_{R}
\end{array}\right] \\
& =\left[\begin{array}{cc}
\left(1-a b u^{2}\right) \mathbf{I}_{V}-c u \mathbf{d} \mathbf{S d}^{*} & 0 \\
u \mathbf{S d}^{*} & \left(1-b^{2} u^{2}\right) \mathbf{I}_{R}
\end{array}\right] .
\end{aligned}
$$

Furthermore, we have

$$
\begin{aligned}
\mathbf{M L} & =\left[\begin{array}{cc}
\left(1-b^{2} u^{2}\right) \mathbf{I}_{V} & 0 \\
u\left(1-b^{2} u^{2}\right) \mathbf{S d}^{*} & -c u \mathbf{S d}^{*} \mathbf{d}-b c u^{2} \mathbf{S d}^{*} \mathbf{d} \mathbf{S}+\left(1-b^{2} u^{2}\right) \mathbf{I}_{R}
\end{array}\right] \\
& =\left[\begin{array}{cc}
\left(1-b^{2} u^{2}\right) \mathbf{I}_{V} & 0 \\
u\left(1-b^{2} u^{2}\right) \mathbf{S d}^{*} & \left(\mathbf{I}_{R}-u\left(c \mathbf{S d}^{*} \mathbf{d}+b \mathbf{S}\right)\right)\left(\mathbf{I}_{R}+u b \mathbf{S}\right)
\end{array}\right] .
\end{aligned}
$$

Here, note that $\mathbf{S}^{2}=\mathbf{I}_{R}$.

For $|t|,|u|$ sufficiently small, we have

$$
\begin{gathered}
\sigma(\Delta(u)), \sigma\left(\left(1-b^{2} t^{2}\right) \mathbf{I}_{V}\right), \sigma\left(\left(1-b^{2} t^{2}\right) \mathbf{I}_{R}\right), \sigma\left(\left(\mathbf{I}_{R}-u\left(c \mathbf{S d}^{*} \mathbf{d}+b \mathbf{S}\right)\right)\left(\mathbf{I}_{R}+u b \mathbf{S}\right)\right) \\
\in B_{1}(1)=\{z \in \mathbf{C}|| z-1 \mid<1\} .
\end{gathered}
$$

Similar to the proof of Proposition 3.8 in [8], $\sigma(\mathbf{L M})$ and $\sigma(\mathbf{M L})$ are contained in $B_{1}(1)$. Thus, $\mathbf{L}$ and $\mathbf{M}$ are invertible, with bounded inverse, for $|t|,|u|$ sufficiently small.

By 1 of Proposition 10, 1 of Proposition 11 and Corollary 12, we have

$$
\begin{aligned}
\operatorname{det}_{\Gamma}(\mathbf{L M}) & =\operatorname{det}_{\Gamma}\left(\left(1-b^{2} u^{2}\right) \mathbf{I}_{V}-c u \mathbf{d} \mathbf{S d}^{*}-b c u^{2} \mathbf{d S}^{2} \mathbf{d}^{*}\right) \operatorname{det}_{\Gamma}\left(\left(1-b^{2} u^{2}\right) \mathbf{I}_{R}\right) \\
& =\left(1-b^{2} u^{2}\right)^{\operatorname{Tr}_{\Gamma}\left(\mathbf{I}_{R}\right)} \operatorname{det}_{\Gamma}\left(\left(1-a b u^{2}\right) \mathbf{I}_{V}-c u \mathbf{d} \mathbf{S d} \mathbf{d}^{*}\right)
\end{aligned}
$$

and

$$
\begin{aligned}
\operatorname{det}_{\Gamma}(\mathbf{M L}) & =\operatorname{det}_{\Gamma}\left(\left(1-b^{2} u^{2}\right) \mathbf{I}_{V}\right) \operatorname{det}_{\Gamma}\left(\mathbf{I}_{R}-u\left(c \mathbf{S d}^{*} \mathbf{d}+b \mathbf{S}\right)\right) \operatorname{det}_{\Gamma}\left(\mathbf{I}_{R}+u b \mathbf{S}\right) \\
& =\left(1-b^{2} u^{2}\right)^{\operatorname{Tr}_{\Gamma}\left(\mathbf{I}_{V}\right)} \operatorname{det}_{\Gamma}\left(\mathbf{I}_{R}-u\left(c \mathbf{S d}^{*} \mathbf{d}+b \mathbf{S}\right)\right) \operatorname{det}_{\Gamma}\left(\mathbf{I}_{R}+u b \mathbf{S}\right)
\end{aligned}
$$


Let an orientation of $G$ be a choice of one oriented edge for each pair of edges in $R(G)$, which is called positively oriented. We denote by $E^{+} G$ the set of positively oriented edges. Moreover, let $E^{-} G:=\left\{e^{-1} \mid e \in E^{+} G\right\}$. An element of $E^{-} G$ is called a negatively oriented. Note that $R(G)=E^{+} G \cup E^{-} G$.

The operator $\mathbf{S}$ maps $\ell^{2}\left(E^{+} G\right)$ to $\ell^{2}\left(E^{-} G\right)$. Then we obtain a representation $\rho$ of $\mathcal{B}\left(\ell^{2}(R(G))\right)$ onto $\mathrm{Mat}_{2} \mathcal{B}\left(\ell^{2}\left(E^{+} G\right)\right)$, under

$$
\rho(\mathbf{S})=\left[\begin{array}{ll}
0 & \mathbf{I} \\
\mathbf{I} & 0
\end{array}\right], \rho\left(\mathbf{I}_{R}\right)=\left[\begin{array}{ll}
\mathbf{I} & 0 \\
0 & \mathbf{I}
\end{array}\right] .
$$

By 1 and 3 of Proposition 11,

$$
\begin{aligned}
\operatorname{det}_{\Gamma}\left(\mathbf{I}_{R}+b u \mathbf{S}\right) & =\operatorname{det}_{\Gamma}\left[\begin{array}{cc}
\mathbf{I} & -b u \mathbf{I} \\
0 & \mathbf{I}
\end{array}\right] \operatorname{det}_{\Gamma}\left[\begin{array}{cc}
\mathbf{I} & b u \mathbf{I} \\
b u \mathbf{I} & \mathbf{I}
\end{array}\right] \\
& =\operatorname{det}_{\Gamma}\left[\begin{array}{cc}
\left(1-b^{2} u^{2}\right) \mathbf{I} & 0 \\
* & \mathbf{I}
\end{array}\right]=\left(1-b^{2} u^{2}\right)^{\frac{1}{2} \operatorname{Tr}_{\Gamma}\left(\mathbf{I}_{R}\right)} .
\end{aligned}
$$

For $|t|,|u|$ sufficiently small, we have

$$
\mathrm{ML}=\mathrm{MLMM}^{-1},
$$

and so, by 2 of Proposition 11,

$$
\operatorname{det}_{\Gamma}(\mathbf{L M})=\operatorname{det}_{\Gamma}(\mathbf{M L}) .
$$

Therefore, it follows that

$$
\begin{gathered}
\left(1-b^{2} u^{2}\right)^{\operatorname{Tr}_{\Gamma}\left(\mathbf{I}_{R}\right)} \operatorname{det}_{\Gamma}\left(\left(1-a b u^{2}\right) \mathbf{I}_{V}-c u \mathbf{d S d}^{*}\right) \\
=\left(1-b^{2} u^{2}\right)^{\frac{1}{2} \operatorname{Tr}_{\Gamma}\left(\mathbf{I}_{R}\right)+\operatorname{Tr}_{\Gamma}\left(\mathbf{I}_{V}\right)} \operatorname{det}_{\Gamma}\left(\mathbf{I}_{R}-u \mathbf{S}\left(c \mathbf{d}^{*} \mathbf{d}+b \mathbf{I}_{R}\right)\right),
\end{gathered}
$$

and so

$$
\begin{gathered}
\operatorname{det}_{\Gamma}\left(\mathbf{I}_{R}-u \mathbf{S C}\right)=\operatorname{det}_{\Gamma}\left(\mathbf{I}_{R}-u \mathbf{S}\left(c \mathbf{d}^{*} \mathbf{d}+b \mathbf{I}_{R}\right)\right) \\
=\left(1-b^{2} u^{2}\right)^{\frac{1}{2} \operatorname{Tr}_{\Gamma}\left(\mathbf{I}_{R}\right)-\operatorname{Tr}_{\Gamma}\left(\mathbf{I}_{V}\right)} \operatorname{det}_{\Gamma}\left(\left(1-a b u^{2}\right) \mathbf{I}_{V}-c u \mathbf{d} \mathbf{S} \mathbf{d}^{*}\right) .
\end{gathered}
$$

Hence the result follows by the definition of $\operatorname{Tr}_{\Gamma}$.

\section{Acknowledgements}

We would like to thank the referee for many useful comments and sincere support. 


\section{References}

[1] H. Bass, The Ihara-Selberg zeta function of a tree lattice, Internat. J. Math., 3: 717-797, 1992.

[2] J. Cheeger and M. Gromov, $L_{2}$-cohomology and group cohomology, Topology, 25: 189-215, 1986.

[3] B. Clair and S. Mokhtari-Sharghi, Zeta functions of discrete groups acting on trees, J. Algebra, 237: 591-620, 2001.

[4] D.Emms, E. R. Hancock, S. Severini andR. C. Wilson, A matrix representation of graphs and its spectrum as a graph invariant, Electron. J. Combin., 13: \#R34, 2006.

[5] B. Fuglede and R. Kadison, Determinant theory in infinite factors, Ann. Math., 55: 520-530, 1952.

[6] R. I. Grigorchuk and A. Żuk, The Ihara zeta function of infinite graphs, the KNS spectral measure and integrable maps, In Random Walk and Geometry, Proc. Workshop (Vienna, 2001), V. A. Kaimanovich et at., eds., pages 141-180. de Gruyter, Berkin, 2004.

[7] D. Guido, T. Isola and M. L. Lapidus, Ihara zeta functions for periodic simple graphs, In $C^{*}$-algebras and elliptic theory II, Edited by D.Burghelea, R.Melrose, A.Mishchenko, E.Troitsky. Trends in Mathematics, pages 103-121. Birkhauser Verlag, Basel, 2008.

[8] D. Guido, T. Isola and M. L. Lapidus, Ihara's zeta function for periodic graphs and its approximation in the amenable case, Journal of Functional Analysis, 255: 1339-1361, 2008.

[9] D. Guido, T. Isola and M. L. Lapidus, Bartholdi zeta function for periodic simple graphs, In Analysis on Graphs and its Applications, Proc. Sympos. Pure Math., 77: 109-122, 2008.

[10] K. Hashimoto, Zeta Functions of Finite Graphs and Representations of $p$-Adic Groups, Adv. Stud. Pure Math. Vol. 15, pages 211-280, Academic Press, New York, 1989.

[11] Yu. Ide, N. Konno, I. Sato, E. Segawa and Y. Shikano, The spectra of the unitary matrix of a general coined quantum walk on a graph, preprint.

[12] Y. Ihara, On discrete subgroups of the two by two projective linear group over $p$-adic fields, J. Math. Soc. Japan, 18: 219-235, 1966.

[13] N. Konno and I. Sato, On the relation between quantum walks and zeta functions, Quantum Inf. Process., 11: 341-349, 2012.

[14] B. Mohar, The spectrum of an infinite graph, Linear Algebra Appl., 48: 245-256, 1982.

[15] B. Mohar and W. Woess, A survey on spectra of infinite graphs, Bull. London Math. Soc., 21: 209-234, 1989. 
[16] P. Ren, T. Aleksic, D. Emms, R. C. Wilson and E. R. Hancock, Quantum walks, Ihara zeta functions and cospectrality in regular graphs, Quantum Inf. Process., 10: 405-417, 2011.

[17] J. -P. Serre, Trees, Springer-Verlag, New York, 1980.

[18] T. Sunada, L-Functions in Geometry and Some Applications, In Lecture Notes in Math., Vol. 1201, pages 266-284. Springer-Verlag, New York, 1986.

[19] T. Sunada, Fundamental Groups and Laplacians (in Japanese), Kinokuniya, Tokyo, 1988. 